\title{
Implementing early supported discharge in stroke care
}

Rebecca J. Fisher

It was with pleasure that my colleagues and I read the recent article by Haussen and Yavagal (Nat. Rev. Neurol. 7, 482-483; 2011), ${ }^{1}$ which discussed our efforts to develop consensus guidelines for early supported discharge (ESD) in stroke care. We are delighted to have the opportunity to offer further thoughts on the key issues raised.

Haussen and Yavagal make an important point about the applicability of the ESD consensus to non-European countries. Although the majority of ESD trials on which the consensus was based were conducted in Europe, valuable contributions were also made by Canadian and Australian ESD trialists. ${ }^{2}$ The ESD consensus clarifies key operational issues associated with delivering ESD, which we feel nicely complement the detailed discussion of postacute clinical intervention featured in the American Heart and American Stroke Associations' statement on interdisciplinary stroke rehabilitation. What remain to be addressed are the "stroke-related costs and cultural perceptions" that Haussen and Yavagal refer to, ${ }^{1}$ which we feel are best explored with qualitative research techniques. We have recently completed a qualitative investigation of the challenges associated with implementing evidence-based ESD services in the UK, exploring the organizational, financial and political issues that impact on delivering the sort of service defined in the ESD consensus. Similar investigations in the USA and other non-European countries would help to identify the barriers to implementing guidelines outlined in the ESD consensus.

A second point raised by Haussen and Yavagal ${ }^{1}$ concerns eligibility for ESD. How are decisions made about which stroke patients can access ESD services and for whom is ESD most beneficial? As outlined by the authors and confirmed in our research, a balance is required between the use of established functional scales (which ensure consistency but can be too restrictive), and the need for clinical reasoning. Our findings highlight the importance of frequent discussion about and reflection on the eligibility criteria that the multidisciplinary team is using. Almost as important as defined criteria are the ways in which individuals involved in delivering stroke care work together, and the need for effective cross-disciplinary and organizational collaboration.

The authors highlight a limitation of the ESD consensus-and current available evidence-in defining the optimal length of the ESD intervention. This issue is crucial, particularly when considering cost implications of ESD. The conclusion drawn from the ESD consensus was that the existence and type of other community stroke services are critical in defining the length of ESD intervention. This conclusion has prompted us to embark on a second activity, involving academics and stroke-service providers and purchasers, to reach consensus on the implementation of longer-term community services for stroke survivors. While ESD is a viable option for patients with mild to moderate stroke, service provision for patients who are not eligible for ESD, or following the ESD intervention, clearly needs to be addressed.

As with the ESD consensus, the aim is that our research on the implementation of community stroke services has wider relevance than a UK setting. With this in mind, we welcome any thoughts on potential opportunities and ways to facilitate this endeavor.

Division of Rehabilitation and Ageing, Community Health Sciences, QMC, B Floor, Medical School, University Park, University of Nottingham, Nottingham NG7 2UH, UK. rebecca.fisher@nottingham.ac.uk

doi:10.1038/nrneurol.2011.128-c1

Competing interests

The author declares no competing interests.

1. Haussen, D. C. \& Yavagal, D. R. Consensus on implementation of early supported discharge. Nat. Rev. Neurol. 7, 482-483 (2011).

2. Fisher, R. J. et al. A consensus on stroke early supported discharge. Stroke 42, 1392-1397 (2011). 